\title{
Congenital hypertrophy of retinal pigment epithelium presenting as incidental finding in a middle aged woman
}

\author{
Harsh Vardhan Singh",*, Iva Rani Kalita ${ }^{2}$, Shubhra Das ${ }^{3}$ \\ ${ }^{1,2}$ Post Graduate Trainee, ${ }^{3}$ Associate Professor, Dept. of Ophthalmology, Regional Institute of Ophthalmology, Guwahati, Assam, \\ India \\ *Corresponding Author: \\ Email: hvsc008@gmail.com
}

\begin{abstract}
Introduction: Retinal Pigment epithelial hypertrophy are uncommon benign, epithelial malformation characterised by typical fundus findings, which is almost always congenital in nature. RPE hypertrophy occurs in three varieties: unifocal, unilateral lesion; multifocal unilateral lesion; and atypical multifocal, bilateral lesion. While the atypical multifocal, bilateral lesions are associated with familial adenomatous polyposis syndrome \& requires systemic evaluation the other two varieties have benign coarse \& doesnot require any intervention.

Case Report: A 42yr Female noticed diminished vision in R/E following trivial trauma with fingernail 14 days back, it was not associated with pain, redness or any other symptoms. On evaluation, BCVA was 6/18p, N6 R/E \& 6/6, N6 L/E; rest anterior segment evaluation was normal. R/E fundus showed focal, 1 DD sized, well-demarcated, hyperpigmented lesion just temporal to fovea with blocked fluorescence in all phase of FFA. OCT \& B-scan showed slightly elevated retinal lesion with optical shadowing. Diagnosis of Retinal pigment epithelial hamartoma (RPEH) was made. Lesion was documented \& followed.

Discussion and Conclusion: RPE hypertrophy are presumed congenital stationary retinal malformation with characteristic ophthalmic, fluorescerin angiographic \& OCT features. These lesions present as asympotomatic incidental findings in childhood or adults with good visual prognosis if located at extrafoveal sites. Gass et al \& shields et al had described various types of RPE malformations \& their characteristic features in FFA \& OCT.

In present case, we have reported a rare case of RPEH \& also demonstrated the role of ancillary tests for diagnosis \& followup.
\end{abstract}

Keywords: Congenital retinal pigment epithelial hypertrophy, RPE hypertrophy, Congenital retinal malformation.

\section{Introduction}

Hypertrophy of the retinal pigment epithelium (RPE) is a rare benign RPE lesion with characteristic fundus findings. RPE hypertrophy is probably a congenital lesion which usually present in late childhood or adult. Three varieties of RPE hypertrophy occur: the unifocal, unilateral lesion; the multifocal, unilateral lesion; \& the atypical multifocal bilateral lesion. Surrounding tissues. Retinal pigment epithelial malformations are the rare conditions with chracteristic clinical features mimicking serious ocular/retinal tumour conditons like retinal adenoma/adenocarcinoma, choroidal melanomas \& malignant melanomas. These RPE tumours are classified into four groups - Congenital Simple Hamartoma of RPE (CSHRPE), Combined hamartoma of Retina \& RPE, combined hypertrophy of RPE, \& adenoma or adenocarcinoma of RPE. ${ }^{1,2}$

The RPE hamartomas are very rare conditions with very few reported cases in the past. Congenital simple retinal hamartomas was first described by Laqua in 1981. ${ }^{3}$ But it was Gass ${ }^{1}$ who coined the term retinal hamartoma in 1989. The largest case series of retinal hamartoma was reported by Shields et $\mathrm{al}^{4}$ in 2003 which included five cases of retinal hamartoma.

Retinal pigment epithelial hamartomas are form of focal congetinal lesion of retinal pigment epithelium characterised by asymptomatic, jet black nodular solitiary lesion frequently involving macular area. ${ }^{1}$ These are very rare conditions with very few reported cases in past. ${ }^{1,3,5}$

Age of Presentation: Laqua $^{3}$ in 1981 had first described this condition \& Gass et $\mathrm{al}^{1}$ in 1989 reported ten cases of RPEH with age of presentation ranging form $8-57$ years.

Characteristics of RPEH Lesions: Focal, Nodular, Jet black full thickness retinal lesion with size ranging from half to one disc diameter(DD), frequently in macular area, not associated with any changes in surrounding sensory retina, rpe or choroid or exudation $\&$ hemorrhages. ${ }^{1}$

FFA \& OCT Findings: FFA present as a blocked fluorescence in both early \& late phase with no associated leakage or vascular malformation. OCT findings includes well demarcated tumour margin with no or mildly elevated tumour surface \& highly reflective retinal tumour with deep optical shadow \& not associated with any epiretinal membrane., ${ }^{4,5}$

Diagnosis: The condition is unilateral $\&$ asymptomatic, and is primarily diagnosed as a incidental finding based on fundus features, along with FFA \& OCT-FMT features to differentiate it from other focal congenital lesions. ${ }^{4-6}$

Course \& Treatment: RPEH is benign non progressive condition \& requires only constant followup but the condition needs to be differentiated from the other life threatening malignant conditions like malignant melanoma or choroidal melanoma or RPE hypertrophy associated with gardener syndrome. 


\section{Case Report}

A 42 year old female presented with a history of trivial trauma to R/E 14 days back followed by sudden onset, non progressive diminusion of vision which she noticed immediately after trauma espescially on closing L/E. DOV was note associated with any pain or redness.

On examination BCVA in R/E was 6/18p, N6 while in L/E 6/6, N6. Anterior segment evaluation were normal in both side. Fundus evaluation right eye showed well demarcated jet black lesion of 1 DD size located just temporal to the fovea (Fig. 1a). On FFA right eye showed hypofluorescence in all phase with hyperfluorescence of temporal margin in late phase (Fig. 1b). OCT evaluation showed slightly elevated lesion with acoustic shadowing without any associated ERM (Fig. 1c). Fundus, OCT FMT \& FFA evaluation were normal for left eye (Fig 2). B-Scan USG of right eye showed nodular echogenic slightly elevated lesion without any ocular shadow (Fig. 3). Other laboratory findings \& GI imaging evaluation were normal.

Based on clinical features, fundus picture, FFA \& OCT features the diagnosis of retinal pigment epithelial hamartoma of right eye was made. Patient was kept under regular followup any change in the retinal lesion \& GI abnormality or polypoidal growths.

On follow-up Evaluation: Retinal lesion size remains unchanged on follow-up at 6 months \& 1 year.
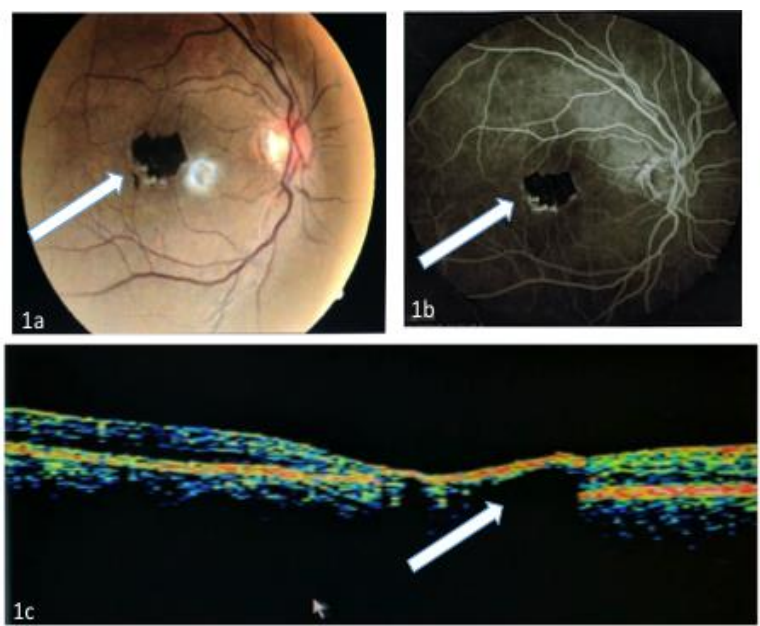
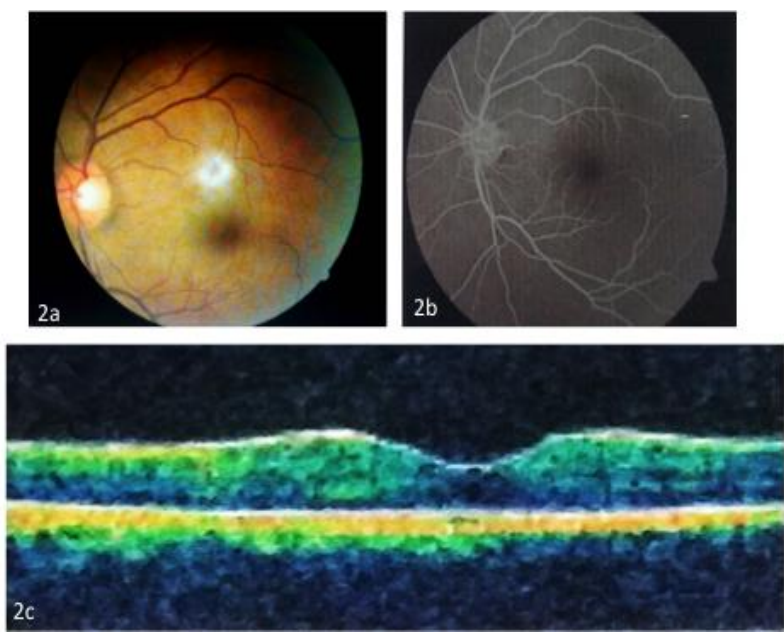

$2 c$

Fig. 1a: Fundus picture of R/E showing 1 DD jet-black discreet lesion temporal to fovea with well demarcated border \& hypopigmentation in temporal margin without any associated abnormal vascularisation or epiretinal membrane; Fig. 1b: FFA of R/E showing hypofluorence area in all phase corresponding with lesion site \& hyper fluorescence of temporal margin in late phase; Fig. 1c: OCT picture of R/E showing slightly raised lesion just temporal to fovea with associated optical shadowing.; Fig. 2a,b,c: Fundus picture, FFA, OCT picture of L/E showing no abnormal changes.

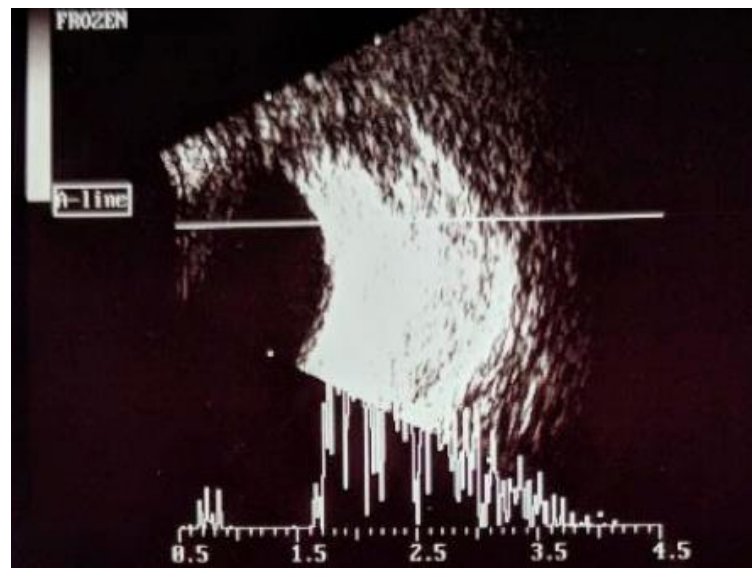

Fig. 3: B-Scan USG of right eye showing nodular echogenic slightly elevated lesion

\section{Discussion}

Tumour or tumour like malformation of RPE are very rare conditions presenting as a diagnostic challenge for the clinicians. Simple Retinal pigment epithelial hamartoma are presumed congenital stationary retinal malformation with characteristic 
ophthalmic, fluorescerin angiographic \& OCF features. These lesions usually present as asympotomatic incidental fundus findings in childhood or young adults with good visual prognosis if located at extrafoveal sites.

Laqua first described the condition of congenital simple RPE hamartoma based on there clinical observation. They reported two cases, 39 year \& 15 year male respectively with incidental finding of focal, well defined, jet black parafoveolar, full retinal lesion with minimally dilated retinal feeding vessels during routine fundus evaluation. ${ }^{3}$

Later in 1989, Gass et al coined the term Retinal pigment epithelial hamartoma (RPEH) \& described four types of focal retinal malformation-RPEH, Combined Retinal pigment epithelial \& retinal hamartoma (CRPE$\mathrm{RH}$ ), Melanocitic naevi of RPE \& Albinotic \& non pigmented naevi of RPE. ${ }^{1}$

Later in 2003 Shields et al reported five case series of RPEH. Later Shield et al \& Sukla et al in 2010 demonstrated the role of OCT as important non invasive diagnostic tool for evaluation of these types of retinal malformation. ${ }^{2,4,5}$

\section{Conclusion}

Because of the rarity of RPEH \& its resemblence with the other malignant pigmented lesions like choridal melanoma, naevus, RPE adenoma, adenocarcinoma or malignant melanoma, good clinical knowledge of these conditions and a carefull long term follow-up to rule out tumour growth or malignant features is needed among ophthalmologists.
In present case report, we reported a very rare case of Simple RPEH diagnosed incidentally in a $42 \mathrm{yr}$ female \& we demonstrated the role of B-scan USG, FFA \& OCT as a important ancillary tests for diagnosis \& follow-up evaluation.

\section{Conflict of Interest: None}

\section{References}

1. Gass JD. Focal Congenital anomalies of the retinal pigment epithelium. Eye (Lond). 1989;3(Pt1):1-18

2. Shields JA, Shields CL. Tumours and related lesions of the retinal pigment epithelium. In: Shields JA, Shields CL, editors. Atlas of Intra Ocular Tumours. Philadelphia: Lippincot Williams \& Wilkins. 1999:287-309.

3. Laqua H. Tumours and tumour-like lesions of the retinal pigment epithelium. Opthalmogica. 1981;183(1):34-38. doi: 10.1159/000309131.

4. Shukla D, Ambatkar S, Jethani J, Kim R. Optical Coherence tomography in presumed congenital simple hamartoma of the retinal pigment epithelium. Am J Opthalmol. 2005;139(5):945-47. doi: 10.1016/j.ajo.2004.11.037.

5. Shields CL, Shields JA, Marr BP, Sperber DE, Gass JD. Congenital simple hamartoma of the retinal pigment epithelium: A study of five cases. Ophthalmology. 2003;110(5):1005-1011. doi: 10.1016/S01616420(03)00087-3.

6. Shields CL, Martin MA, Kartza EC, Shields JA. Optical Coherence tomography of congenital simple hamartoma of the retinal pigment epithelium. Retina. 2004;24(2):327-328. 